\title{
A behavioural change intervention study for the prevention of childhood obesity in South Africa: protocol for a randomized controlled trial
}

\author{
Natisha Dukhi ${ }^{*}$ (D), Benn Sartorius ${ }^{2,3}$ and Myra Taylor $^{2}$
}

\begin{abstract}
Background: South Africa is currently undergoing a nutrition transition, and overweight and obesity is on the increase in South African children. Urbanization and other health determinants have led to reduced physical activity and unhealthy eating that have increased the risk of adverse chronic health conditions. This study aims to provide evidence of the effectiveness of a school-based intervention study that targets diet and physical activity for the prevention of child and adolescent overweight and/or obesity.

Methods: We will employ a mixed method study design which is divided into two phases. Phase 1, namely the qualitative elicitation research phase will inform the development of the quantitative intervention phase (phase 2), consisting of a cluster-randomized trial, based on input from key stakeholders. The study will be undertaken in 16 government-funded primary schools in the iLembe district of KwaZulu-Natal, South Africa. The study will target learners in Grades 4 and 7, their parents, Life Orientation educators, school principals and members of school governing bodies. Assessment for the primary objective (BMI Z scores), and the secondary objectives (change in knowledge, attitudes and behaviours regarding diet and physical activity) in both study arms will be conducted at baseline in March 2020 and at the end of the study in October 2020.

Discussion: The study will be a novel combined mixed methods/RCT design that focuses on diet, physical activity school and family-based interventions in the context of rapidly increasing overweight and obesity prevalence in KwaZulu-Natal. To encourage behaviour change and management of malnutrition, education including diet and physical activity, is an important strategy that must be considered. Nutrition education extends beyond the dissemination of food information; it includes addressing the needs of participants, empowers and encourages decision-making and choice of foods, change in nutrition attitudes, beliefs and influences based on resources available and within cultural boundaries.
\end{abstract}

Trial registration: Pan African Clinical Trial Registry PACTR201711002699153. Protocol registered on 16 November 2017.

Keywords: Children, Adolescents, School, Diet, Nutrition, Physical activity, Overweight, Obesity, Cluster randomized controlled trial

\footnotetext{
*Correspondence: doctordukhi@gmail.com; NDukhi@hsrc.ac.za

${ }^{1}$ Social Aspects of Public Health, Human Sciences Research Council, Cape

Town, South Africa

Full list of author information is available at the end of the article
}

(c) The Author(s). 2020 Open Access This article is distributed under the terms of the Creative Commons Attribution 4.0 International License (http://creativecommons.org/licenses/by/4.0/), which permits unrestricted use, distribution, and reproduction in any medium, provided you give appropriate credit to the original author(s) and the source, provide a link to the Creative Commons license, and indicate if changes were made. The Creative Commons Public Domain Dedication waiver (http://creativecommons.org/publicdomain/zero/1.0/) applies to the data made available in this article, unless otherwise stated. 


\section{Background}

Contributing to the over nutrition epidemic, an estimated 340 million children and adolescents aged 5-19 years were overweight or obese in 2016 [1]. The World Health Organization (WHO) global nutrition targets for 2025, especially target 4 , has a specific focus on preventing an increase in childhood overweight [2]. The rapid increase in overweight and obesity in children and adolescents, largely due to modifiable risk factors such as unhealthy eating and physical inactivity, is strongly influenced by the physical environment, as well as cultural, social and economic factors [3]. It is estimated that by the year 2020, lifestylerelated non-communicable diseases (NCDs) will account for $60 \%$ of the burden of disease, and $70 \%$ of deaths worldwide, if adequate health promotion intervention programmes are not established. To reduce this chronic disease burden, modification of one's lifestyle, promotion of prevention measures against NCDs and improved dietary habits and physical activity are important $[4,5]$. Furthermore, a cause for public health concern is the increasing trend of child and adolescent weight gain as they transition into adulthood. Adolescents, 10-19 years of age, as defined by the WHO, are targeted for interventions as it is during this growth and development period that health attitudes, beliefs and behaviours are established and continue into adulthood [3]. The adolescent years have been referred to as the "tipping years" by Hanvey et al., [6], where co-morbidities such as high blood pressure, diabetes, stroke, cardiovascular disease and cancer may appear as short term health consequences [7].

In an effort to reverse and stop the increased trend of overweight and obesity, children should be brought up in a health promoting environment where instead of traditionally following the choices of others they can make informed choices about their health [3]. The school is viewed as an ideal health-promoting environment that can develop children and adolescents physically, emotionally and socially [8]. Literature on school obesity interventions exists, and while some literature that specifically focuses on diet and physical activity exists, such tend to focus on anthropometric measures such as body mass index (BMI) only, self-efficacy and some positive evaluations and outcomes [9-11]. In South Africa, there have been minimal obesity intervention studies, with some focusing on physical activity only, and nutrition interventions having either a food questionnaire to identify food choices or BMI measurements only. The HealthKick study, comprising of diet and physical activity interventions is the only known study in South Africa that aimed to address the prevention of overweight in children and reduce the risk of chronic diseases such as type 2 diabetes. This study identified significant improvement in self-efficacy and nutrition knowledge but not in children's nutrition behaviour. Limitations of the study included lack of parent participation, as well as the authors suggesting that creating a healthier food environment within and around the school was important [12]. The study, in addition to other international studies, has been helpful in assisting in the development of the iLembe School Physical Activity and Nutrition (i-SPAN) study. The i-SPAN study identified the strength and weaknesses of South African studies and while these studies were implemented with some success in low-income areas, literature for school-based diet and physical activity interventions is still limited in the African setting [13-15].

Therefore, there is a need to develop and implement interventions that are specific for lifestyle diseases and conditions that target the child groups relevant to South Africa. Such interventions may be difficult to conduct and sustain due to a lack of funding and resources. Thus, the i-SPAN study has been developed and will be conducted in the poverty-challenged iLembe District in the province of KwaZulu-Natal in South Africa. The elicitation research phase will inform the development of an intervention phase that will design, implement and assess the effectiveness of school-based interventions that focus on diet and physical activity in children and adolescents in Grades 4 and 7 (aged 9-15 years). It is during these years that most girls (8-13 years) and boys (10-15 years) enter puberty, reaching a growth peak that results in a healthy BMI and weight [16].

\section{Trial aims and objectives}

The aim of this cluster randomized controlled trial (RCT) is to provide evidence of the effectiveness of diet and physical activity interventions conducted in schools in preventing overweight and obesity in children and adolescents.

\section{Specific objectives \\ Phase one: elicitation research}

1. To assess the health promotion status of children and adolescents in the iLembe district schools (what children know about diet and exercise, and their diet and physical activities), explore and understand the school children's attitudes, cultural beliefs, vision and ideas regarding obesity, and identify the extent to which health promotion has been utilized in the schools regarding diet and physical activity.

2. To identify facilitating and constraining factors for health promotion (diet and physical activity) in the schools in iLembe district according to the key stakeholders such as Department of Basic Education (DoBE), Department of Health (DoH), Department of Sports and Recreation and other stakeholders such as the Health Promotion Coordinators, District Nutrition Coordinator, School Health Teams. 
3. To design and implement diet and physical activity school-based interventions to reduce obesity in school children.

\section{Phase two: intervention}

4. To assess the effectiveness of the i-SPAN study in children and adolescents aged 9-15 years by comparing the intervention and control arms at baseline and at the end of the study regarding:

a. BMI Z scores.

b. Proportion of children and adolescents classified as overweight and obese.

c. Knowledge, attitude and behaviours regarding diet and physical activity.

5. To describe and evaluate the outcomes of the interventions for scientific dissemination.

\section{Methods}

The protocol has been developed using the Standard Protocol Items: Recommendations for Interventional Trials (SPIRIT) guidelines [17] (Additional file 1).

\section{Design}

The study is a cluster randomized controlled trial, with both quantitative and qualitative components, to determine the effectiveness of the i-SPAN study to prevent overweight and/or obesity in children and adolescents (Fig. 1).

\section{Participants and recruitment process}

The participants of the study will include children in grade four (aged 9-11 years) and grade seven (aged 12-15 years), as well as parents/primary caregivers, Life Orientation (LO) educators, principals, members of the community, the latter through the school governing body (SGB). When the data has been collected meetings will be held with the SGBs (from whom permission will be required prior to the study) to inform them of the results and get their input on the way forward. They will also be the link with the wider community. Other stakeholders will include DoBE, DoH, Sports and Recreation, Health Promotion Coordinators, the District Nutrition Coordinator and School Health Teams in iLembe district, KwaZulu-Natal. The study setting will be primary schools in iLembe district.

A complete list of all schools with grade four and seven children in iLembe district will be provided by the Provincial Department of Basic Education. The list includes over 400 primary schools from which 16 schools will be selected randomly with probability proportional to size (PPS). There will be four schools from each of the four sub-districts, which will be randomly assigned into the two study arms (control $n=8$, intervention $\mathrm{n}=$ 8). Two schools will receive the intervention and two schools will serve as controls in each of the four subdistricts. All children and adolescents in grade four and seven attending these schools and their parents/primary caregiver will be invited to participate in the study. Children and adolescents must provide assent in writing via the consent forms. Parents/caregiver must also provide consent for participation in the study for the children/ adolescents and themselves (Additional file 2). South African schools are arranged into quintiles determined by the National Treasury, based on scores reflective of the poverty levels where a community is situated. The DoBE uses these poverty classifications that range from quintiles $1-5$, where quintile five is the least povertystricken and one is the most poverty-challenged quintile, to classify schools. Most of the primary schools in iLembe district are from quintiles 1 and 2 , hence it is important to understand the daily challenges these communities face regarding housing, socio-economic status, employment, as well as health outcomes as these are regarded as schools with low socio-economic status and exempt from school fees [18].

\section{Sample size/power calculation}

The school is the unit of the study. The power/sample size calculation was performed using a test for two means in a cluster randomized design function implemented in PASS 12 [19]. A power calculation indicated that with 8 schools in each arm and 68 participants per cluster, the study would have $90 \%$ power (1- $\beta$ ) to detect an underlying medium standardized difference between the means (effect size) of 0.3 following the intervention, at a two-sided $5 \%$ significance level $(\alpha)$, assuming an intra-class correlation coefficient (ICC) of 0.05 or $5 \%$ [20, 21].

\section{Randomization}

The school is the unit of the study. A biostatistician who does not have direct contact with study participants will generate the random allocation sequences using a computerized random number generator. After the inclusion of each cluster, the allocation of that particular cluster will be provided to the study coordinator. There will be no crossover between study arms.

\section{Inclusion/exclusion criteria}

The inclusion criteria include: a) Children and adolescents in grades four and seven (aged 9-15 years). b) Parent/primary caregiver of the participating children/ adolescents. c) Children and adolescents with physical disabilities will be included in the study but excluded from the anthropometry.

\section{Study components and implementation}

The study will consist of two phases: Phase 1 is a baseline elicitation phase using a qualitative paradigm, whereby the 


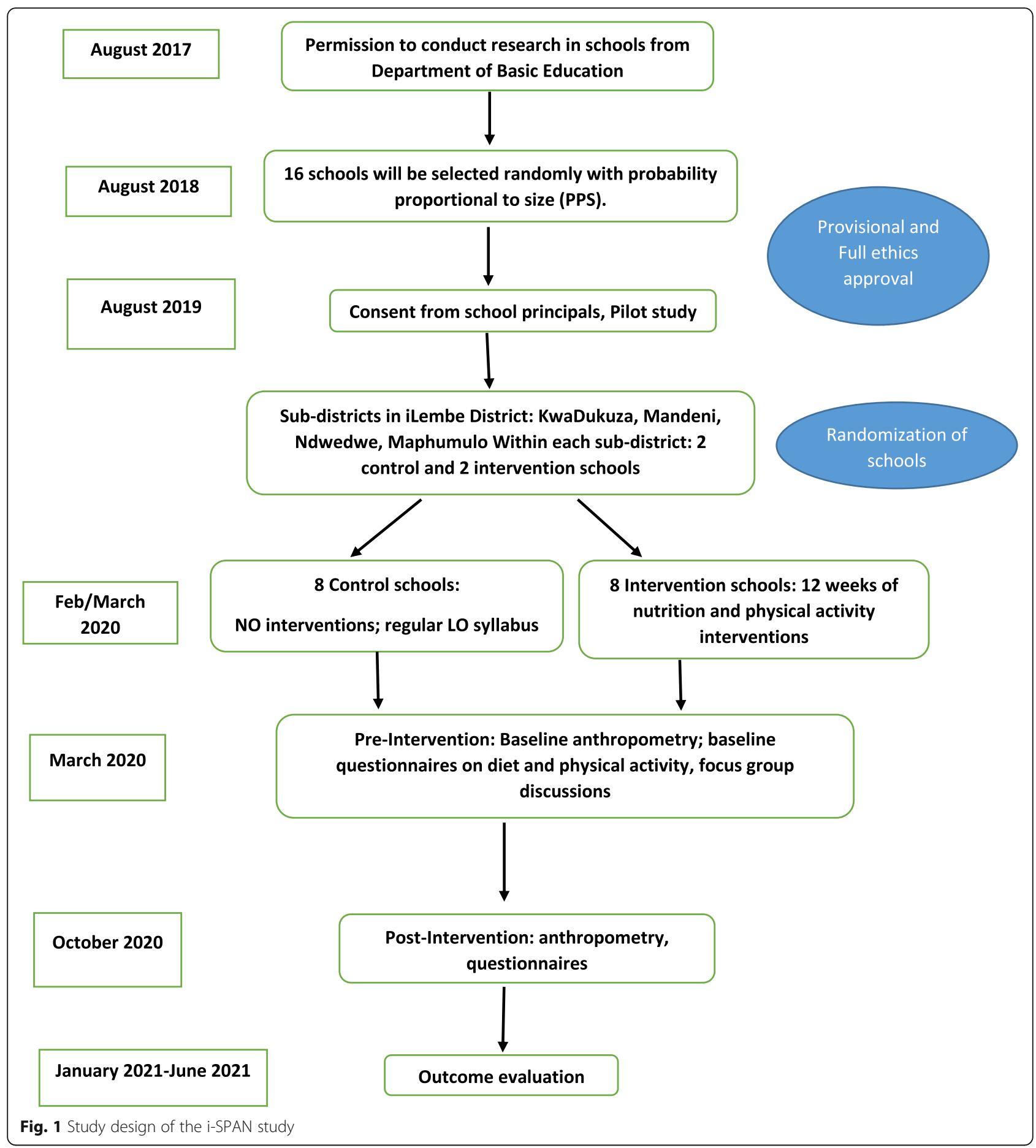

formative assessment will inform the development of the intervention for use in phase 2. A thorough search and review of available scientific literature will be carried out to determine what is currently being done regarding learners, particularly in respect to health promotion concerning physical activity and dietary habits of school-going learners in South Africa and specifically in iLembe district,
KwaZulu-Natal. During the elicitation phase focus group discussions (FGDs) will be conducted with selected members of the SGB, as well as selected children and adolescents in grades four and seven to explore current practices at each school. In-depth interviews will be conducted with principals regarding nutrition and PA at school. Based on the information obtained from the elicitation research 
phase, a plan for the intervention phase based on the Social Cognitive Theory (SCT) framework [22] will be developed and implemented. During the intervention phase structured questionnaires will be given to the children and adolescents in grades four and seven, the LO educators and the parents/primary caregivers of the participating children and adolescents to determine their understanding, knowledge, attitudes and behaviours regarding diet and physical activity at baseline and at the end of the study.

\section{Training}

Fieldworkers will be recruited for the study and training will continue in 2020. A training manual will be compiled by the principal investigator and co-investigators of the study, who will also facilitate the training. During the training fieldworkers will be given an in-depth background about the significance of the study and the scope of malnutrition in the South African and the global context. They will be carefully trained on the study instruments that will be used such as FGDs and structured interview questions, administering of the intervention questionnaires and the measuring of height and weight in children and adolescents. Fieldworkers must be fluent in isiZulu and English. They will also be trained on their role in the study, as well as their interactions with members of the school, community and stakeholders such traditional leaders. Once the aims of the study and the fieldworker role is understood they will be given the information and consent forms in both English and isiZulu to role play as these have been selected as the two mediums of communication in the schools. During role play various scenarios will be created to allow fieldworkers to be adequately prepared in their approach to the schools in the study. Further training will be conducted with fieldworkers whereby they are introduced to the equipment that will be used for the study. Repeated training and a pilot phase will focus on achieving inter- and intra-rater reliability of measures.

\section{Pilot study}

Prior to the study, the survey instruments will be piloted in one school that will not be included in the study by the researcher/fieldworkers. It will be important to test the questionnaire and measurement instruments to eliminate any ambiguity in the questions asked in the questionnaire and to correct any difficulties experienced before the main study. The pilot study will follow the same procedures as the main study but use different schools. The data from the pilot study will not be included in the main study. Confidentiality of the participants will be maintained at all times throughout the pilot and main study. The participant's name or any personal details relating to the participants will not be mentioned in any publication, report or meetings where results of the researcher are being presented.

\section{Data collection instruments and techniques}

Table 1 below identifies and describes the measures to be assessed and techniques utilized in the i-SPAN study.

\section{Behavioural outcomes}

We believe there are two important behaviour change outcomes that can be achieved from the study, i.e. healthy food choices and increased food variations, and increased physical activity participation during school hours. Table 2 below describes the two behavioural outcomes and the anticipated change based on the SCT model [22].

\section{Mediating and moderating variables}

The i-SPAN learner questionnaire will be used to assess any potential mediators, as part of the process evaluation, to identify any possible processes that may mediate diet and physical activity changes. The questionnaire consists of different sections that are able to assess knowledge, attitudes and beliefs, as well as the mediating behaviours of the child and parent that may affect diet and physical activity levels. Possible moderating variables such as sex, weight status and healthy lifestyle behaviours will be obtained from the baseline data of the study. The questionnaires will be administered during the classroom LO lessons by the fieldworkers to ensure that the learners understand the questions.

\section{Process evaluation}

This phase will seek to identify the acceptability and impact of the interventions on the learners, educators and parents. It will also explore the extent to which the health promotion toolkit will be utilised in the intervention schools. Various factors such as facilitating and constraining factors to implement toolkit at the schools, the implementation process, times and durations of implementation will be taken into account. Therefore, to conduct the process evaluation a mixed methods approach will be utilised.

\section{Planned data collection}

The data will be de-identified and available for secondary analyses for student projects. Electronic data quality will be ensured by checking completeness of required variables throughout the data collection process, and will comprise: number of participants correspond to number of questionnaires completed. Absenteeism will be recorded. For data analysis and results, all personal identifiers of participants will be removed during reporting phases. All data will be handled and coded only by the principal investigator and co-investigators who will serve as a data monitoring committee for the study. The principal investigator will also be responsible for auditing trial conduct, as well as any adverse events during the trial. Reports will be presented and made available to the various stakeholders mentioned previously and UKZN to assist in managing and preventing overweight and obesity in childhood. The 


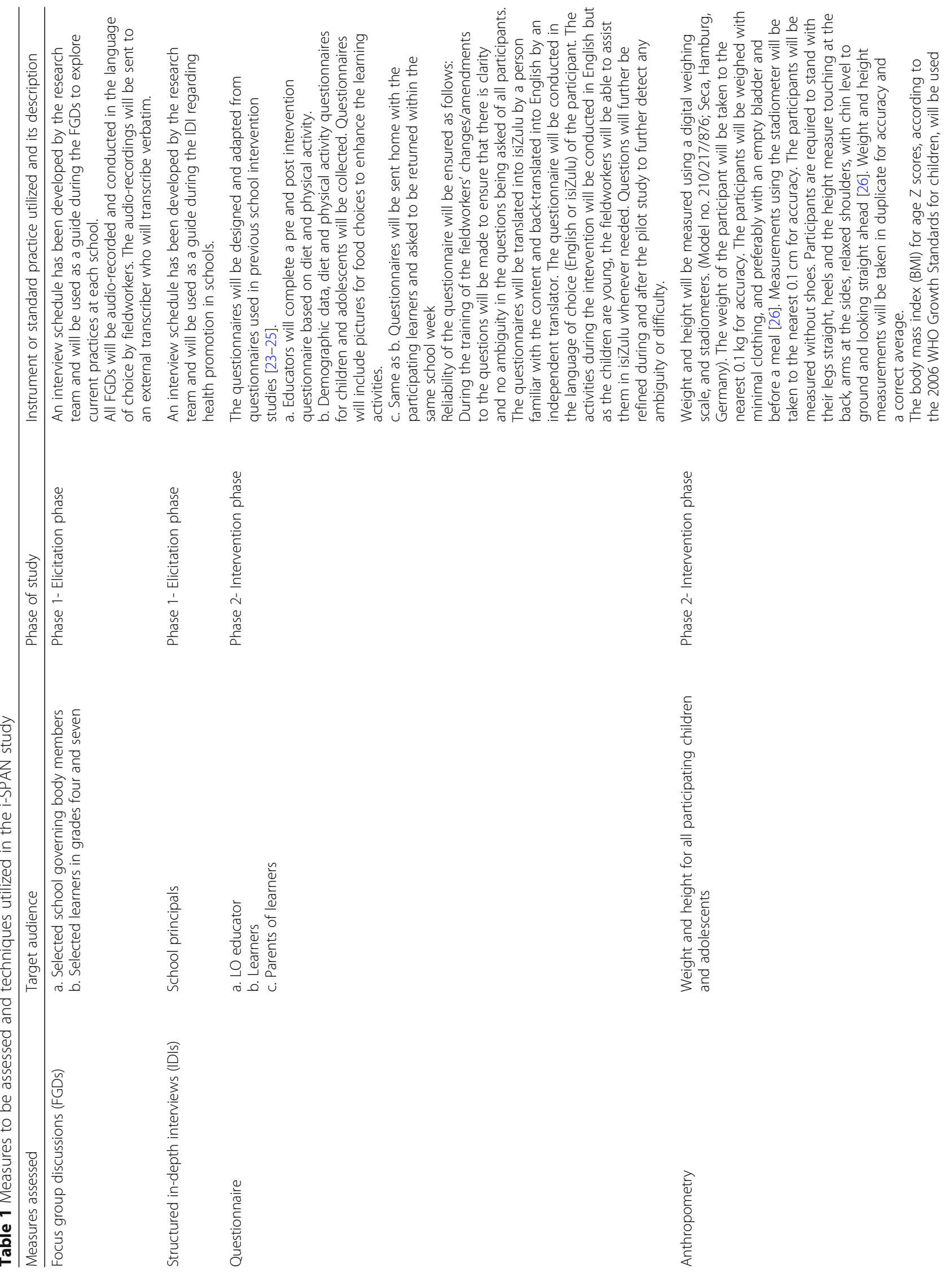




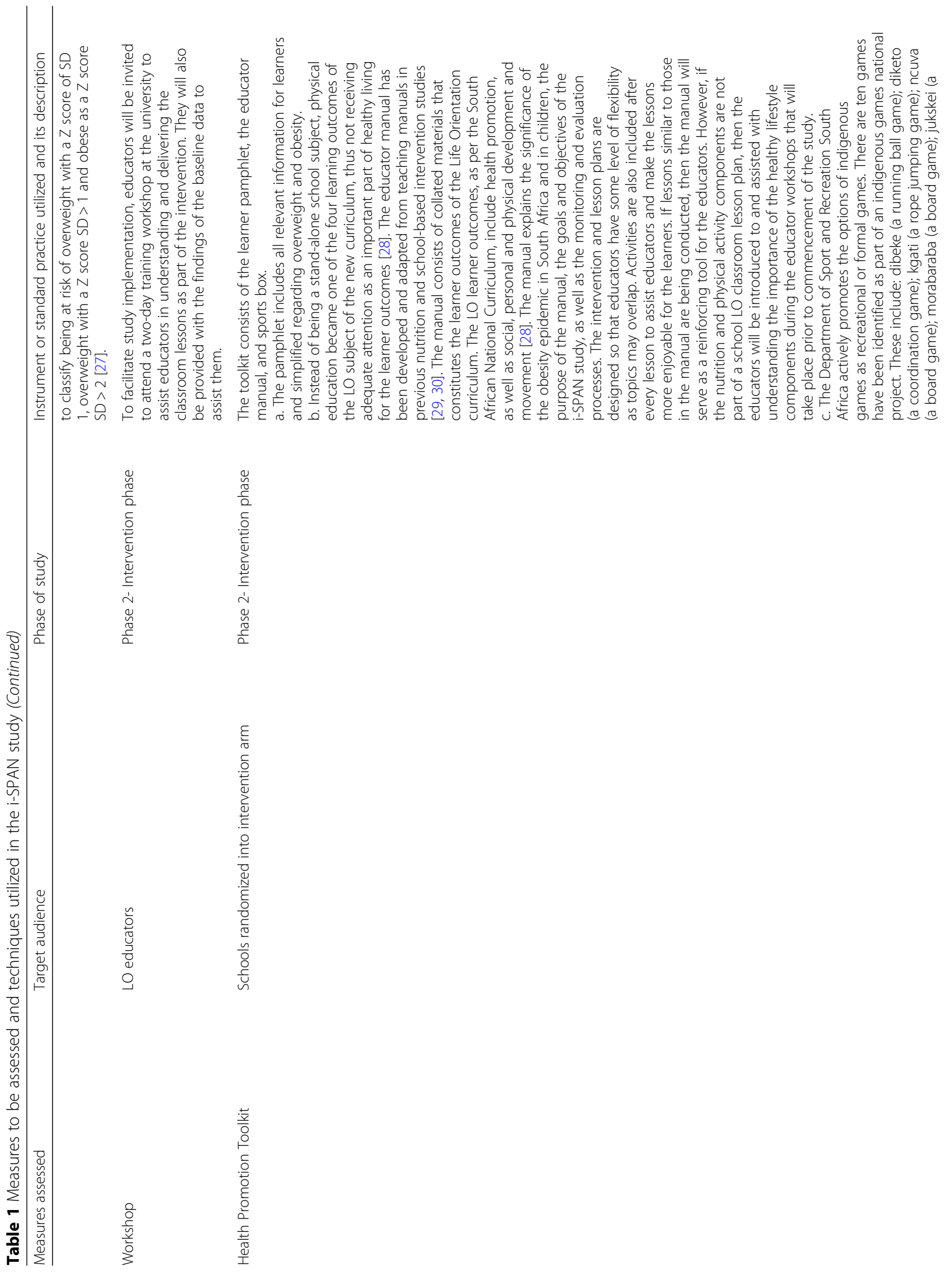




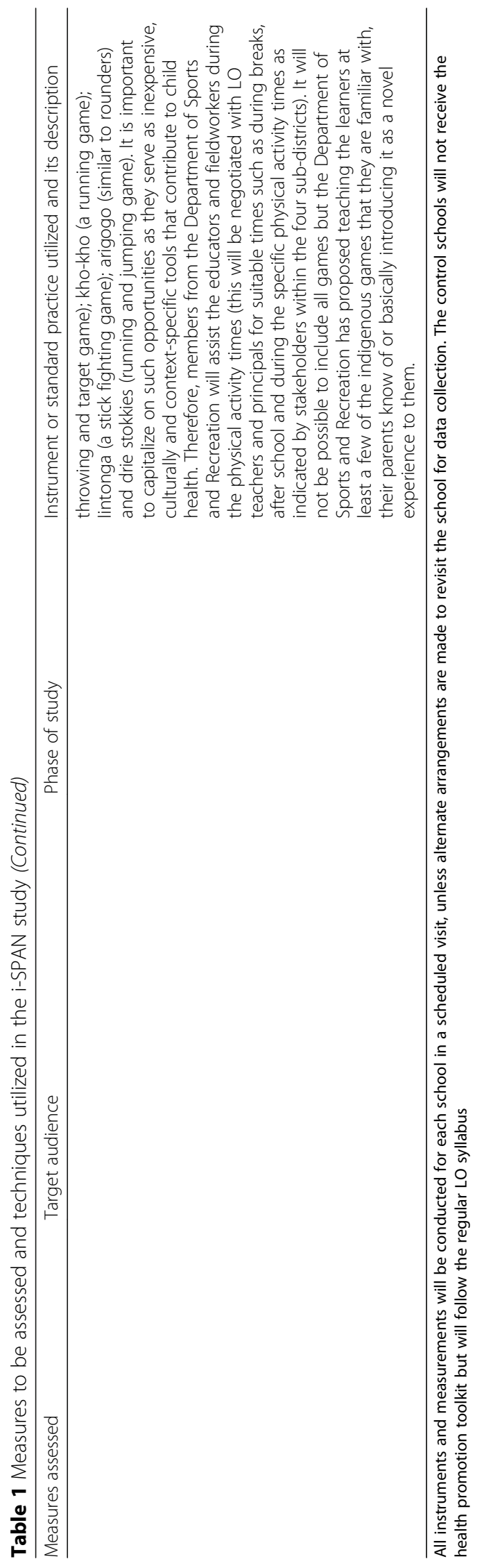




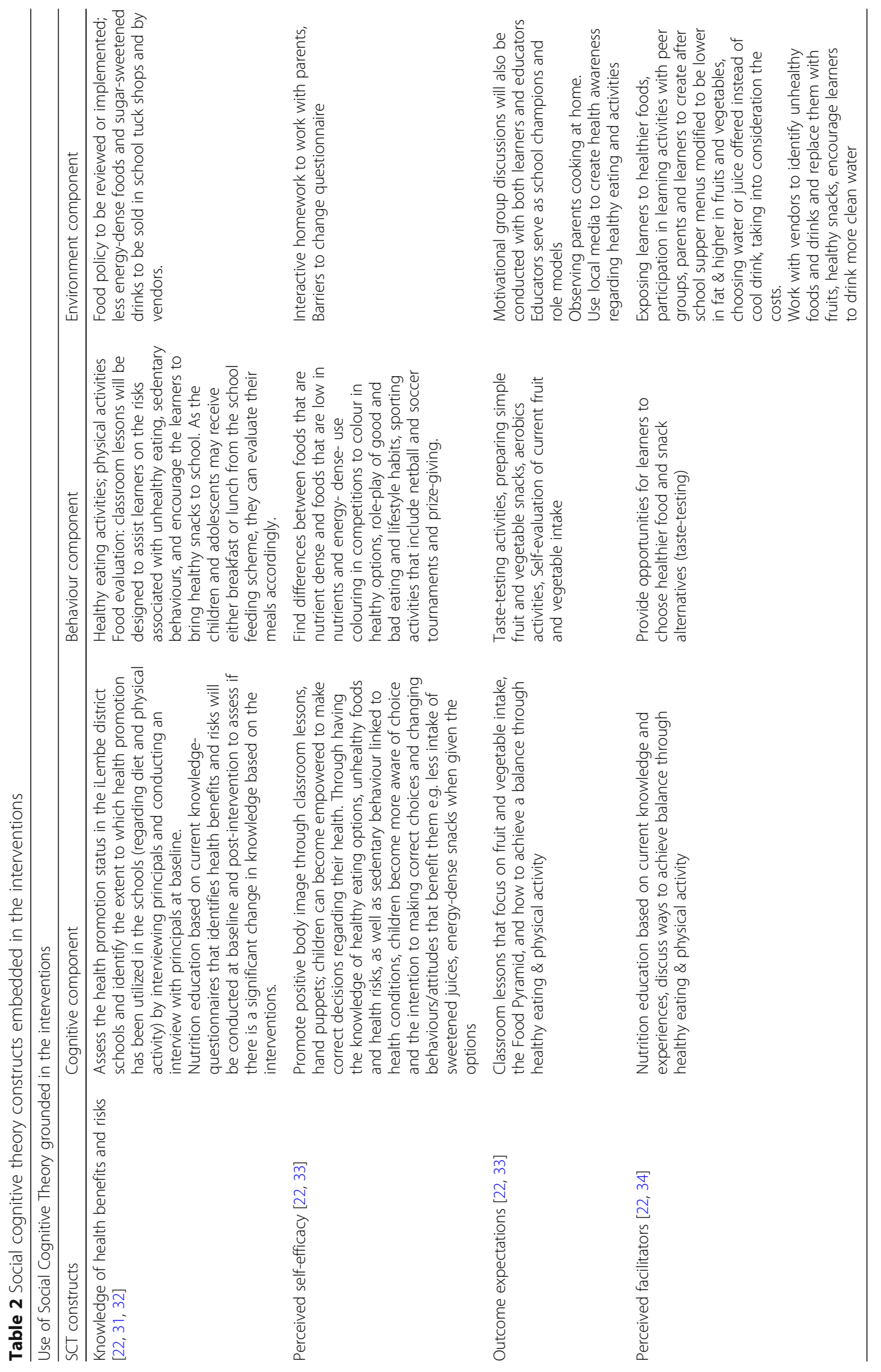


data will also serve to assist the community i.e. iLembe district in managing and preventing overweight and obesity in children and adolescents. Findings of the study will also be disseminated through conferences and peerreviewed publications. During the survey if learners are identified as overweight or obese, will be referred for appropriate care to local health facilities as per Department of Health levels of care.

\section{Planned statistical analysis Descriptive and analytical statistics}

The use of weight and height, BMI calculations, completed questionnaires, arising from the interventions will use both descriptive and inferential statistics to analyse the data. To ensure succession of cluster randomization, the presence of any significant differences between the control and intervention cluster for the baseline characteristics of the participants such as sex, age, etc., will be examined using bivariate mixed effect regressions to account sufficiently for the clustered design. Data will be processed and analysed using Stata 13.0 SE. Mixed effects using generalized linear and logistic modelling approaches will be used to compare continuous and binary endpoints respectively among students in schools in intervention and control arms of the study. A within school random effect will be incorporated to account for correlated (repeated) measurements within each school to correctly estimate the significance when comparing preand post-intervention periods. Outcome variables include change in BMI at baseline and end of study, dietary intake, time spent in sedentary behaviours, diet and physical activity self-perception and practices, including overall knowledge and attitude regarding diet and physical activitysignificant levels and stratified analyses.

\section{Qualitative analysis}

All transcribed data from the FGDs and in-depth interviews will be managed using NVIVO8. The data will be imported to the NVIVO8 program, linked to memos where coding will occur. Topics and concepts will be coded i.e. to capture information about happenings, ideas or specific topics, and nodes will be created to mark relevant information to be analysed or searched [35]. Thereafter, data will be manually analysed for emerging common themes.

\section{Discussion}

To our knowledge, this is the only other RCT to be conducted in South Africa, and the first in KwaZulu-Natal, that includes diet and physical activity school-based interventions. To encourage behaviour change and management of malnutrition nutrition, education (that includes diet and physical activity) is an important strategy that must be considered [36]. Nutrition education extends beyond the dissemination of food information; it includes addressing the needs of children and adolescents, parents and the community, empowers and encourages decisionmaking and choice of foods, encourages change in attitudes, beliefs and influences based on resources available and the needs of individuals, as well as improving and promoting the nutrition knowledge within cultural boundaries $[37,38]$. Nutrition education serves to not only impart important nutrition information and health messages but rather involves the participants to engage in activities and/ or interventions that will improve nutrition [39]. If the iSPAN study is effective, this RCT will be implemented to other schools, including the study control schools in iLembe district and outside of iLembe as part of the prevention and management of childhood obesity in KwaZulu-Natal.

\section{Supplementary information}

Supplementary information accompanies this paper at https://doi.org/10. 1186/s12889-020-8272-1.

Additional file 1. The Standard Protocol Items: Recommendations for Interventional Trials (SPIRIT) guidelines

Additional file 2. Information sheet and participant consent

\section{Abbreviations}

BMI: Body mass index; BREC: Biomedical Research Ethics Committee; DoBE: Department of Basic Education; DoH: Department of Health; FGD: Focus group discussion; i-SPAN: iLembe School Physical Activity and Nutrition; LO: Life Orientation; NCDs: Non-communicable diseases; PPS: Probability proportional to size; RCT: randomized controlled trial; SCT: Social Cognitive Theory; SPIRIT: Standard Protocol Items:

Recommendations for Interventional Trials; UKZN: University of KwaZuluNatal; WHO: World Health Organization

Acknowledgements

Not applicable.

Authors' contributions

ND is the Co-PI of the study and drafted the manuscript. All authors contributed to the protocol development and MT as PI provided overall protocol development expertise. BS provided statistical expertise. All authors read, amended and approved the final manuscript.

\section{Funding}

This research did not receive any specific grant from funding agencies in the public, commercial, or not-for-profit sectors.

Availability of data and materials Not applicable.

\section{Ethics approval and consent to participate} Ethics approval was obtained from the University of KwaZulu-Natal Biomedical Research Ethics Committee (BREC) (reference number: BFC555/17). Informed written consent will be obtained from the learner participants and their parents, including all other study participants.

\section{Consent for publication}

Once recruitment commences consent for publication will be sought from all participants.

\section{Competing interests}

The authors declare that they have no competing interests.

\section{Author details}

${ }^{1}$ Social Aspects of Public Health, Human Sciences Research Council, Cape

Town, South Africa. ${ }^{2}$ Discipline of Public Health Medicine, School of Nursing 
and Public Health, University of KwaZulu-Natal, Durban, South Africa. ${ }^{3}$ Faculty of Tropical and Infectious Diseases, London School of Hygiene and Tropical Medicine, London, UK

Received: 23 July 2019 Accepted: 24 January 2020

Published online: 04 February 2020

\section{References}

1. World Health Organization (WHO). Obesity and overweight Factsheet. 2017. http://www.who.int/mediacentre/factsheets/fs311/en/dc.gov/healthyweight/ effects/index.html Accessed 31 Oct 2017

2. World Health Organization (WHO). e-Library of Evidence for Nutrition Actions (eLENA). Interventions by global target. 2017. http://www.who.int/ elena/global-targets/en/ Accessed 31 Oct 2017.

3. World Health Organization (WHO). Addressing the socioeconomic determinants of healthy eating habits and physical activity levels among adolescents. 2006. www.euro.who.int/document/e89375.pdf Accessed 31 Oct 2017.

4. Abegunde DO, Mathers CD, Adam T, Ortegon M, Strong K. The burden and costs of chronic diseases in low-income and middle-income countries. Lancet. 2007:370:1929-38.

5. Sarrafzadegan N, Kelishadi R, Esmaillzadeh A, Mohammadifard N, Rabiei K, Roohafza H, Azadbakht L, Bahonar A, Sadri G, Amani A, Heidari S, Malekafzali $H$. Do lifestyle interventions work in developing countries? Findings from the Isfahan Healthy Heart Program in the Islamic Republic of Iran. Bull World Health Organ. 2009:87:39-50.

6. Hanvey AN, Mensah FK, Clifford SA, Wake M. Adolescent cardiovascular functional and structural outcomes of growth trajectories from infancy: prospective community-based study. Child Obes. 2017;13(2):154-63.

7. Franks $P$, Hanson RL, Knowler W, Sievers M, Bennett PH, Looker HC. Childhood obesity, other cardiovascular risk factors, and premature death. N Engl J Med. 2010;362:485-93.

8. Aldinger C, Zhang X, Liu L, Pan X, Yu S, Jones J, Kass J. Changes in attitudes, knowledge, and behaviour associated with implementing a comprehensive school health program in a province of China. Health Educ Res. 2008;23(6):1049-67.

9. Luepker RV, Perry CL, McKinlay SM, Nader PR, Parcel GS, Stone EJ, Webber LS, Elder JP, Feldman HA, Johnson CC, et al. Outcomes of a field trial to improve children's dietary patterns and physical activity. The child and adolescent trial for cardiovascular health. CATCH collaborative group. JAMA. 1996;275(1):768-76.

10. Day ME, Strange KS, McKay HA, Naylor PJ. Action schools! BC-healthy eating: effects of a whole-school model to modifying eating behaviours of elementary school children. Can J Public Health. 2008:99(1):328-31.

11. Stevens J, Story M, Ring K, Murray DM, Cornell CE, Gittelsohn J. The impact of the pathways intervention on psychosocial variables related to diet and physical activity in American Indian schoolchildren. Prev Med. 2003;37(2):70-9.

12. de Villiers A, Steyn NP, Draper CE, Hill J, Dalais L, Fourie J, Lombard C, Barkhuizen G, Lambert EV. Implementation of the HealthKick intervention in primary schools in low-income settings in the Western Cape Province, South Africa: a process evaluation. BMC Public Health. 2015;15(818):1-12.

13. Draper CE, Kolbe-Alexander TL, Lambert EV. Factors contributing to the success of a physical activity-based health promotion programme: a retrospective evaluation. J Phys Activ Health. 2009;6(1):578-88.

14. Naidoo $R$, Coopoo $Y$, Lambert EV, Draper CE. The impact of a primary schoolbased nutrition and physical activity intervention on learners in KwaZulu-Natal, South Africa: a pilot study. S Afr J Sports Med. 2009:21(1):10-5.

15. Draper CE, de Kock L, Grimsrud AT, Rudolph M, Nemutandani MS, KolbeAlexander TL, Lambert EV. Evaluation of the implementation of a school-based physical activity intervention in Alexandra township, South Africa. S Afr J Sports Med. 2010:22(1):12-9.

16. Soliman A, De Sanctis $V$, Elalaily R. Nutrition and pubertal development. Indian J Endocrinol Metab. 2014:18(1):S39-47.

17. Chan A-W, Tetzlaff JM, Altman DG, Laupacis A, Gøtzsche PC, Krleža-Jerić K, Hróbjartsson A, Mann H, Dickersin K, Berlin J, Doré C, Parulekar W, Summerskill W, Groves T, Schulz K, Sox H, Rockhold FW, Rennie D, Moher D. SPIRIT 2013 statement: defining standard protocol items for clinical trials. Ann Intern Med. 2013:158:200-7.

18. Pauw K. Profile of the North West Province: demographics, poverty, inequality and unemployment. Provided Project Background Paper. 2005;1(6):1-9.

19. NCSS Statistical Software. PASS 15. 2017. www.ncss.com Accessed 31 Oct 2017.

20. Donner A, Klar N. Statistical considerations in the design and analysis of community intervention trials. J Clin Epidemiol. 1996;49(4):435-9.
21. Donner A, Klar N. Design and analysis of cluster randomization trials in Health Research. London: Arnold; 2000.

22. Bandura A. Health promotion by social cognitive means. Health Educ Behav. 2004;31(2):143-64.

23. Coordinated Approach to Child Health (CATCH). Programs and Resources 2014-2017. 2017. https://catch.org/ Accessed 31 Oct 2017.

24. Katzmarzyk PT, Barreira TV, Broyles ST, Champagne CM, Chaput JP, Fogelholm M, Hu G, Johnson WD, Kuriyan R, Kurpad A, Lambert EV, Maher C, Maia J, Matsudo V, Olds T, Onywera V, Sarmiento OL, Standage M, Tremblay MS, Tudor-Locke C, Zhao P, Church TS. The international study of childhood obesity, lifestyle and the environment (ISCOLE): design and methods. BMC Public Health. 2013;13(900):1-13.

25. Central Health Education Unit, Centre for Health Protection, Department of Health, The Government of the Hong Kong Special Administrative Region. Assessment of Dietary Pattern in Primary Schools 2008. 2012. http://www. chp.gov.hk/files/pdf/Report_part1_English.pdf Accessed 31 Oct 2017.

26. Cogill, B. Anthropometric Indicators Measurement Guide. 2003. http://www. fantaproject.org Accessed 31 Oct 2017.

27. World Health Organization (WHO). WHO Child Growth Standards: Length/ height-for-age, Weight-for-age, Weight-for-length, Weight-for-height and Body mass index-for-age: Methods and Development. Geneva: World Health Organization; 2006. http://www.who.int/childgrowth/standards/technical_ report/en/ Accessed 31 Oct 2017

28. Van Deventer K. Perspectives of teachers on the implementation of life orientation in grades R-11 from selected Western cape schools. S Afr J Educ 2009:29(1):127-45

29. Department of Health -Abu Dhabi (HAAD) Schools for Health Program. Eat Right Get Active Health Campaign. 2017. https://schoolsforhealth.haad.ae/ health-campaigns/healthy-eating.aspx Accessed 31 Oct 2017.

30. Food and Agriculture Organization of the United Nations (FAO). Eating well for good health. 2017. http://www.fao.org/docrep/017/i3261e/i3261e00.html Accessed 31 Oct 2017

31. DiClemente R, Salazar LF, Crosby RA. Health behavior theory for public health: principles, foundations, and applications. Burlington: Jones and Bartlett Learning; 2013.

32. Safdie M, Cargo M, Richard L, Lévesque L. An ecological and theoretical deconstruction of a school-based obesity prevention program in Mexico. Int J Behav Nutr Phys Act. 2014;11:103.

33. Bandura A. (Ed): social foundations of thought and action: a social cognitive theory. Englewood Cliffs: Prentice-Hall; 1986.

34. Bandura A. Social cognitive theory: an Agentic perspective. Annu Rev Psychol. 2001;52:1-26.

35. Walsh M. Teaching qualitative analysis using QSR NVivo. Qual Rep. 2003;8(2):251-6.

36. Western Cape Government. Integrated Nutrition Programme. A foundation for life. South Africa: Western Cape Government; 2013. https://www.westerncape. gov.za/service/integrated-nutrition-programme Accessed 31 Oct 2017

37. Department of Health South Africa (DoH). Guidelines for nutrition interventions at health facilities to manage and prevent child malnutrition: Department of Health South Africa; 2003. http://www.up.ac.za/media/ shared/62/ZP_Files/implementation_guidelines_for_nutrition_interventions at_health.zp70745.pdf Accessed 31 Oct 2017

38. Food and Agriculture Organization (FAO) of the United Nations (UN). Nutrition Education for the public is essential. Global Forum on Food Security and Nutrition Policies and Strategies (FSN Forum) Brief. 2008 http://www.fao.org/fsn/ Accessed 31 Oct 2017.

39. Contento IR, Randel JS, Basch CE. Review and analysis of Evaluation Measures in Nutrition Education Intervention Research (NEIR). J Nutr Educ Behav. 2002;34:2-25

\section{Publisher's Note}

Springer Nature remains neutral with regard to jurisdictional claims in published maps and institutional affiliations. 